\title{
Black Holes and Abelian Symmetry Breaking
}

\author{
Javier Chagoya ${ }^{1}$, Gustavo $\mathrm{Niz}^{2}$, Gianmassimo Tasinato ${ }^{1}$ \\ (1) Department of Physics, Swansea University, Swansea, SA2 8PP, U.K. \\ (2) Departamento de Física, Universidad de Guanajuato - DCI, C.P. 37150, León, Guanajuato, México.
}

\begin{abstract}
Black hole configurations offer insights on the non-linear aspects of gravitational theories, and can suggest testable predictions for modifications of General Relativity. In this work, we examine exact black hole configurations in vector-tensor theories, originally proposed to explain dark energy by breaking the Abelian symmetry with a non-minimal coupling of the vector to gravity. We are able to evade the no-go theorems by Bekenstein on the existence of regular black holes in vector-tensor theories with Proca mass terms, and exhibit regular black hole solutions with a profile for the longitudinal vector polarization, characterised by an additional charge. We analytically find the most general static, spherically symmetric black hole solutions with and without a cosmological constant, and study in some detail their features, such as how the geometry depends on the vector charges. We also include angular momentum, and find solutions describing slowly-rotating black holes. Finally, we extend some of these solutions to higher dimensions.
\end{abstract}




\section{Contents}

1 Introduction $\quad 1$

2 System under consideration $\quad 3$

3 Static, spherically symmetric, charged black hole solutions $\quad 4$

3.1 Asymptotically flat configurations in absence of a cosmological constant 5

$\begin{array}{ll}3.2 & \text { Configurations including a cosmological constant }\end{array}$

4 Slowly rotating black hole configurations $r$

$\begin{array}{llr}5 & \text { Higher dimensional solutions } & 9\end{array}$

$\begin{array}{llr}6 & \text { Discussion } & 9\end{array}$

\section{Introduction}

The physics of black holes provides fundamental insights for understanding the nature of gravity in the non-linear regime. The direct detection by Advanced LIGO of gravitational waves produced by the GW150914 event [1] opens new opportunities for direct tests of black hole properties, and more generally, to test the theory gravity. At the dawn of this new observational era, it is important to theoretically understand how much the physics of black holes depends on the theory of gravity under consideration.

While classic results have been established in the past decades, there are open questions on black holes within Einstein gravity coupled to other fields, or in modified gravity scenarios. The no-hair conjecture states that black holes are characterised by at most three quantities: mass, angular momentum, and electromagnetic charge. Including a scalar field does not normally add a supplementary conserved charge to the black hole configuration. See e.g. [2] for a recent review on the status of the conjecture, what has been proved so far, and possible counterexamples to it.

If we modify our theory of gravity, Birkhoff theorem does not necessarily apply, and black hole configurations may result different than those in GR. Recently, there has been an increasing interest of going beyond GR, considering new scalar-tensor or vector-tensor theories of gravity aimed to describe dark energy or inflation [3]. The most interesting among these approaches exhibit a phenomenon called screening mechanism. Light fields can drive present day cosmic acceleration, and at the same time hide their presence thanks to screening mechanisms, which suppress fifth force effects and satisfy stringent Solar system constraints. See e.g. [4] for a review on this subject. One of the most well studied realizations of screening is the Vainshtein mechanism, first proposed in [5] in the context of Fierz-Pauli massive gravity: the effect of graviton scalar polarization is concealed by its own non-linear self derivative-interactions. A broad class of theories exhibiting Vainsthein screening involve Galileons or their extensions [6], and more generally one can construct an EFT from the Horndeski scalar-tensor set-up which captures most of the Vainshtein screening terms of other well known theories [7]. Models exhibiting (Vainshtein) screening exploit non-linear effects in the scalar sector, but have been so far mostly analysed only in the weak gravity regime where the tensor sector is treated linearly - and around spherically symmetric sources (see [8-10] for exceptions though). See [11] for consequences of the Vainshtein mechanism in astrophysics. 
The study of black holes in theories of modified gravity which exhibit a screening phenomenon is interesting for various reasons. On one hand, it provides configurations where tensor non-linearities are important, hence new arenas where screening effects can be tested, possibly in new ways. On the other hand, novel examples of black hole solutions in consistent theories where gravity is nonminimally coupled with additional fields, can shed further light on the generality of the black hole no-hair conjecture, and suggest new testable features of black hole physics.

Important results have been obtained so far for explicit examples of black holes in scalar-tensor theories with screening properties, as Galileon or Horndeski systems. In [12], the authors derive a no-go theorem against the existence of static configurations in theories equipped by appropriate shift symmetries. Ways out to this conclusion have been found in $[13,14]$, violating some of the hypothesis of the no-go theorem, and furnishing explicit black hole configurations. Alternatively, simple black hole solutions have been determined in [15] by considering time dependent scalar configurations. Today, there are already several results on black holes within Horndeski-like theories, including generalisations of the original solutions, and studies of possible applications to astrophysics; see for example [2, 16-19] for recent reviews.

Black hole solutions in vector-tensor theories of gravity have been less investigated recently, although it is an equally interesting subject with good physical motivations. When coupled with gravity, Abelian vector fields provide a charge, and an associated long range force, to black hole configurations. Here we focus on theories which break Abelian symmetry, in which the vector longitudinal polarization becomes dynamical. We investigate whether black hole solutions can exist in these theories. We have to start facing a powerful no-go theorem: Bekenstein proved that if the Abelian symmetry is broken by an explicit vector mass term, then regular black holes do not exist [20], [21], [22] (see also [23] for a generalization). We find ways out considering the class of vector theories breaking Abelian symmetry, introduced in [24-26], which do not need to rely on mass terms. The theories we consider are related to Galileons, are free of ghostly extra modes, and are known to exhibit screening effects in appropriate cases [24, 27]. They can be consistently covariantized when coupled with gravity [24, 26], leading to second order equations of motion (see also [28] for further developments on these or related theories). The no-go theorems of [20] are circumvented by the fact that, in the cases that we consider, Abelian symmetry is broken only by appropriate couplings with gravity. We determine exact, regular black hole solutions for the specific vector-tensor set-up that we consider, with interesting properties that we summarize here:

- In absence of a bare cosmological constant, the requirement of asymptotical flatness uniquely fixes the coupling between vector and gravity. We are able to explicitly solve all the field equations, and find the most general static spherically symmetric solution for the system. Its geometry coincides with the Schwarzschild black hole, but with both transverse and longitudinal polarizations turned on for the vector. We examine the properties of the system, and on which extent our configuration violates the no-hair conjecture.

- When including a bare cosmological constant, all the field equations can again be solved exactly, and we determine the most general, static spherically symmetric solution. We investigate in detail the resulting geometry, which depends both on the value of the cosmological constant, and ona charge controlling the profile of the vector's longitudinal polarization.

- We find and analyse exact solutions corresponding to slowly-rotating black holes, in absence of a cosmological constant. In this case, the geometry corresponds to the slowly-rotating Kerr metric, and several of the vector components have to be turned on in order to solve the field equations.

- We extend the static, asymptotically flat solutions to higher dimensions, finding results qualitatively similar to the four dimensional case. 


\section{System under consideration}

We consider the following action

$$
S=\int d^{4} x \sqrt{-g}\left\{\frac{M_{\mathrm{Pl}}^{2}}{2} R-\frac{1}{4} F^{2}-\Lambda+\beta\left[\left(D_{\mu} A^{\mu}\right)^{2}-D_{\mu} A_{\nu} D^{\nu} A^{\mu}-\frac{1}{2} A^{2} R\right]\right\} .
$$

This theory describes Einstein gravity with a cosmological constant, supplemented with standard, gauge invariant kinetic term for a vector field $A_{\mu}$ (whose field strength is $F_{\mu \nu}=\nabla_{\mu} A_{\nu}-$ $\nabla_{\nu} A_{\mu}$ ), plus an additional contribution weighted by a dimensionless constant $\beta$, breaking the Abelian gauge symmetry by coupling the vector $A_{\mu}$ with gravity. The first two terms inside the square parenthesis, depending on the derivatives of the vector potential, are a total derivative in flat space when gravity is turned off. To appreciate better this fact, after an integration by parts (and neglecting boundary terms), we can re-express the action $S$ as

$$
S=\int d^{4} x \sqrt{-g}\left[\frac{M_{\mathrm{Pl}}^{2}}{2} R-\frac{1}{4} F^{2}-\Lambda+\beta G_{\mu \nu} A^{\mu} A^{\nu}\right],
$$

with $G_{\mu \nu}$ the Einstein tensor. The expression (2.2) makes manifest that we are breaking the gauge symmetry $A_{\mu} \rightarrow A_{\mu}+\partial_{\mu} \xi$ due to a direct, non-minimal coupling of the Maxwell potential $A_{\mu}$ with gravity. The theory propagates five degrees of freedom: two helicity-2 modes (tensors) associated with the gravity sector; two helicity-1 modes (often called vectors), associated with the transverse components of the the vector potential $A_{\mu}^{T}$; and one helicity-0 (scalar), the longitudinal component of the vector. The dynamics of the longitudinal vector polarization is turned on by the coupling proportional to $\beta$. Due to specific couplings of gravity to the vector, no additional sixth mode propagates $[24,26,29]$. On the other hand, the sign of the kinetic terms of the physical modes - in particular the vector longitudinal polarization - depends on the background under consideration.

This is the simplest among the theories studied in [24, 26] which break the vector Abelian symmetry, in such a way to avoid introducing a ghostly sixth mode. Nevertheless, it has a sufficiently rich structure for our purposes. The field equations derived from (2.1) are (indices between brackets are symmetrized)

$$
\begin{aligned}
\frac{M_{\mathrm{Pl}}^{2}}{2} G_{\mu \nu}= & \frac{1}{2}\left[F_{\mu \rho} F_{\nu}{ }^{\rho}-\frac{1}{4} g_{\mu \nu} F^{2}\right]-\beta\left[\frac{1}{2} g_{\mu \nu}\left(D_{\alpha} A^{\alpha}\right)^{2}-2 A_{(\mu} D_{\nu)} D^{\alpha} A_{\alpha}+g_{\mu \nu} A_{\alpha} D^{\alpha} D^{\beta} A_{\beta}\right. \\
& +\frac{1}{2} g_{\mu \nu} D_{\alpha} A_{\beta} D^{\beta} A^{\alpha}-2 D^{\alpha} A_{(\mu} D_{\nu)} A_{\alpha}+D_{\alpha}\left(A_{(\nu} D_{\mu)} A^{\alpha}+A_{(\mu} D^{\alpha} A_{\nu)}-A^{\alpha} D_{(\mu} A_{\nu)}\right) \\
& \left.-\frac{1}{2}\left(A^{2} G_{\mu \nu}+A_{\mu} A_{\nu} R-D_{\mu} D_{\nu} A^{2}+g_{\mu \nu} \square A^{2}\right)\right] . \\
D^{\mu} F_{\mu \nu}= & -2 \beta G_{\mu \nu} A^{\mu} .
\end{aligned}
$$

The right hand side of eq (2.3) corresponds to the energy momentum tensor (EMT) for this theory. The Abelian symmetry is only broken in curved space-times. Actually, in the weak gravity regime, where $G_{\mu \nu}$ is negligible, the Abelian symmetry is conserved to a very good approximation. Similar theories with vector fields coupled with curvature - although not with the Einstein tensor, the only coupling that ensures the absence of a ghostly sixth mode - have been used in cosmology (see e.g. [30, 31]); here we investigate the existence and properties of black holes.

Back in 1971, Bekenstein proved the non-existence of black hole solutions in theories breaking Abelian symmetry by a mass term [20]. In few words, Bekenstein's argument starts with noticing that Proca's mass term leads to a non-vanishing 'right hand side' (RHS) for the vector equation (the equivalent of our equation (2.4)). When static configurations are considered, this RHS term 
does not allow one to turn on time-like and space-like components $A_{0}$ and $A_{i}$ simultaneously, since this would break the time-reversal symmetry which characterises the system. If we analyse electrically charged configurations, with $A_{0}$ turned on, this implies that $A_{i}$ must vanish; then, a clever consideration based on the properties of a certain integral allows Bekenstein to conclude that regular black holes cannot exist in this theory, without even having to start to solve the field equations. See also [32] for additional explanations.

In our work, we break the Abelian symmetry not by a mass term, but by a direct coupling with gravity, leading to the vector field equation (2.4). This is how we avoid Bekenstein's argument, since - as we will explicitly show - the longitudinal mode of the vector field is coupled to gravity in such a way that the relevant part of the right hand side of equation (2.4) automatically vanishes for our static configurations. This fact implies that we can consistently turn on both the $A_{0}$ and $A_{i}$ components, without violating the time-reversal symmetry of the system, hence avoiding Bekenstein's arguments. Moreover, we find that the additional Abelian symmetry breaking contribution in the action (2.2) have important effects in determining the global properties of the spherically symmetric configuration. The energy momentum tensor in (2.3), associated with the gauge field, backreacts on the geometry so to modify the Reissner-Nordström charged black hole solution ${ }^{1}$.

\section{Static, spherically symmetric, charged black hole solutions}

In this section we study static, spherically symmetric solutions for the system described by action (2.1). Our Ansatz for the metric and the vector field configuration is

$$
\begin{aligned}
d s^{2} & =-f(r) d t^{2}+h(r)^{-1} d r^{2}+r^{2} d \theta^{2}+r^{2} \sin ^{2} \theta d \varphi^{2}, \\
A_{\mu} & =\left(A_{0}(r), \pi(r), 0,0\right) .
\end{aligned}
$$

This is the most general static, spherically symmetric Ansatz allowing for an electrically charged configuration, associated with $A_{0}(r)$. Notice that we also allow for a non-trivial profile of $\pi(r)$; this is a key difference with respect to the usual Reissner Nordström black hole. A non-trivial profile for $\pi(r)$ is associated with the vector longitudinal polarization, which has an important role in our scenario that breaks Abelian symmetry.

Given our field Ansatz, the equations of motion for the system can be expressed as

$$
\begin{aligned}
0= & \beta \pi\left[h-f\left(f+r f^{\prime}\right)^{-1}\right], \\
0= & 2 f\left[M_{\mathrm{Pl}}^{2}(h-1)+r^{2} \Lambda\right]+h r^{2}{A_{0}^{\prime}}^{2}+2 M_{\mathrm{Pl}}^{2} r h f^{\prime} \\
& +\beta\left[2 A_{0}^{2}(h-1)+2 f h(3 h-1) \pi^{2}+8 A_{0} h r A_{0}^{\prime}-2 A_{0}^{2} h r \frac{f^{\prime}}{f}+6 h^{2} \pi^{2} r f^{\prime}\right], \\
0= & 2 f\left[M_{\mathrm{Pl}}^{2}\left(1-h-r h^{\prime}\right)-r^{2} \Lambda\right]-h r^{2} A_{0}^{\prime 2} \\
& +\beta\left[2 A_{0}^{2}\left(h-1+r h^{\prime}\right)-2 f h \pi\left(\pi\left(h+1+3 r h^{\prime}\right)+4 h r \pi^{\prime}\right)\right], \\
0= & 4 A_{0} \beta\left(h-1+r h^{\prime}\right)-r\left[A_{0}^{\prime}\left(h r \frac{f^{\prime}}{f}-4 h-r h^{\prime}\right)-2 h r A_{0}^{\prime \prime}\right] .
\end{aligned}
$$

where a prime ' indicates derivative along the radial direction. When $\beta \neq 0$ and the scalar profile is non-vanishing, the first equation determines $h$ only in terms of $f$. As a consequence, the Ricci scalar can be generally expressed as

$$
R=\frac{\left(2 f-r f^{\prime}\right)\left(2 f^{\prime}+r f^{\prime \prime}\right)}{2 r\left(f+r f^{\prime}\right)^{2}}
$$

\footnotetext{
1 As an aside comment, there is another way to avoid Bekenstein's arguments by considering asymptotically Lifshitz configurations. Indeed, exact black hole configurations for massive vector fields have been found in these theories [33]. Intriguingly, these solutions have some properties in common with ours, as we will comment later.
} 
Therefore, if $f$ asymptotically scales with some power of $r, f \sim r^{n}$ for large $r$ (with $n \neq-1$ ) then the Ricci scalar vanishes at infinity, $R \sim 1 / r^{2}$.

We start discussing solutions in absence of cosmological constant.

\subsection{Asymptotically flat configurations in absence of a cosmological constant}

When $\beta=0$, standard steps lead to Reissner-Nordström configuration as a unique electrically charged, spherically symmetric solution for the field equations

$$
\begin{aligned}
f & =h=1-\frac{2 M}{r}+\frac{Q^{2}}{2 M_{\mathrm{Pl}}^{2} r^{2}}, \\
A_{0} & =\frac{Q}{r}+P \\
\pi & =0 .
\end{aligned}
$$

This configuration is asymptotically flat. Since gauge symmetry is preserved, the vector longitudinal mode profile $\pi(r)$ is not physical and can be switched off. In the previous formulae, $M$ is the black hole mass and $Q$ is the black hole electric charge. Notice that the additional integration constant $P$ in the vector profile has no physical effects, and can be set to zero thanks to gauge symmetry. On the other hand, the electric charge $Q$ affects the metric introducing a $Q / r^{2}$ correction to the Schwarzschild metric; the geometry 'feels' the electric charge. See [34] for a textbook discussion, including an extension to magnetically charged configurations.

However, when $\beta \neq 0$, the structure of the solution is very different. We require that the metric asymptotically approaches Minkowski space. This means that we focus on the large $r$ limit of the configuration, where we can approximate $f=h=1$. Substituting $f=h=1$ in equations (3.3a)-(3.3d), we find that they can be satisfied for a non-trivial profile for $A_{0}$, but only if

$$
\beta=\frac{1}{4} .
$$

So the requirement of approaching a flat space asympotically, singles out a unique value for the coupling constant ${ }^{2}$. Remarkably, after making the choice (3.8), we can return back to the set of equations (3.3a)-(3.3d) and find that a simple manipulation of them ${ }^{3}$ provides a unique static solution, which turns out to be asymptotically flat. The most general solution reads

$$
\begin{aligned}
f & =h=1-\frac{2 M}{r} \\
A_{0} & =\frac{Q}{r}+P \\
\pi & =\frac{\sqrt{Q^{2}+2 P Q r+2 M P^{2} r}}{r-2 M}
\end{aligned}
$$

where $M$ is the black hole mass, while $Q$ and $P$ indicate charge parameters. This unique static solution has various interesting properties, which can be summarised as follows:

- The geometry is described by the Schwarzschild solution characterised by $M$, and it is independent of the charges $Q$ and $P$. The Abelian symmetry breaking self-interactions contribute

\footnotetext{
${ }^{2}$ We also checked that requiring more generic asymptotic behaviours for $f$ and $h$ - for example demanding a power-law - also fixes (different) values for $\beta$. However, it is then not possible to find simple solutions for the complete equations for all values of $r$.

${ }^{3}$ We can solve eq (3.3a) for $h$, and substitute the results in the remaining equations. (3.3c) provides a simple differential equation for $A_{0}$, with (3.10) as unique solution. Substuting this profile in eqs (3.3b), (3.3d), we find simple linear differential equations leading to $(3.9),(3.11)$ as general solutions. There is no solution for $\pi(r)=0$.
} 
to the energy momentum tensor in such a way to exactly compensate for the contributions of the vector charges to the geometry. In some sense, the vector self-interactions are able to screen the vector charges $Q$ and $P$ from the geometry, which is insensitive to the presence of the vector. Such a behaviour was also found in scalar-tensor black holes [15] for a similar system, leading to so-called stealthy Schwarzschild configurations. Also, in asymptotically Lifshitz systems [33] with massive vectors the geometry does not depend on the vector profile.

- Since the geometry corresponds to a Schwarzschild configuration, the corresponding Einstein tensor vanishes, implying that the right hand side of the vector field equation (2.4) is zero. As we explained in Section 2, this avoids Bekenstein's arguments [20], since we can have both $A_{0}$ and $\pi$ switched on without breaking the time reversal symmetry, allowing us to find a regular black hole configuration in an Abelian symmetry breaking theory.

- Each of the curvature invariants, and the components of the EMT, are well behaved for $r>0$. No essential singularities are induced on the system by the new vector interactions, besides the singularity at the origin, covered by the Schwarzschild horizon. The singularity at $r=2 M$ in the scalar profile $\pi(r)$ is only apparent - analogous to the apparent singularity in the horizon of the geometry - and does not appear when computing the components of the EMT.

- The vector profile $A_{0}(r)$ and the scalar profile $\pi(r)$ depend on two independent integration constants, $Q$ and $P$. The profile for $A_{0}(r)$ leads to a long range electric field sourced by the black hole, identical to the Reissner-Nordström configuration. While, as we have seen, the parameter $P$ has no physical implications for the Reissner-Nordström black hole, in this case it is a charge controlling the scalar profile $\pi(r)$ (although not the geometry). Depending on the presence or not of $P$, the scalar profile $\pi(r)$ scales as $1 / \sqrt{r}$ or $1 / r$ at large distances. Notice also that we can turn off the charge $Q$ - so to switch off the long range electric field and leave the charge $P$, characterising a non-trivial scalar profile, turned on in the presence of a black hole mass.

In this sense, $P$ represents an additional charge for the configuration, besides mass and electric charge. However this charge is not 'seen' by applying the standard Gauss law - since the field $\pi$ does not contribute to $F_{\mu \nu}$ - hence strictly speaking this configuration does not violate the no-hair conjecture. On the other hand, the scalar profile might be probed by some test object which couples to the vector field $A_{\mu}$ in a way which does not respect the Abelian symmetry. It would be interesting to analyse specific systems which can probe in this way the longitudinal component of $\pi$. This is an interesting, but more model dependent issue that we leave for future work.

We explicitly checked that our configuration is stable under perturbations that are spherically symmetric (but time dependent), provided that $Q$ and $P$ are small. In the next subsection, we discuss how the addition of a cosmological constant drastically changes the properties of the geometry.

\subsection{Configurations including a cosmological constant}

We now consider how including a cosmological constant affects our configuration. Interestingly, the equations of motion determining a static, spherically symmetric configuration, can again be solved straightforwardly in the case of $\beta=1 / 4$. Making this choice for this parameter, with a procedure 
analogous to the one of Section 3.1, we find that the most general solution of our set of equations is

$$
\begin{aligned}
f & =1-\frac{2 M}{r}+\frac{4 r^{2} \Lambda_{P}}{3}+\frac{4}{5} r^{4} \Lambda_{P}^{2}, \\
h & =f\left(1+2 r^{2} \Lambda_{P}\right)^{-2}, \\
A_{0} & =\frac{Q}{r}+P\left(1+\frac{2}{3} r^{2} \Lambda_{P}\right), \\
\pi & =\sqrt{\frac{A_{0}^{2}}{f h}-\frac{P^{2}\left(1+2 r^{2} \Lambda_{P}\right)-8 M_{\mathrm{Pl}}^{2} r^{2} \Lambda_{P}}{h}} \\
& =\frac{1+2 r^{2} \Lambda_{P}}{\sqrt{f}}\left[\frac{\left(3 Q+r P\left(3+2 r^{2} \Lambda_{P}\right)\right)^{2}}{9 r^{2} f}-P^{2}\left(1+2 r^{2} \Lambda_{P}\right)+8 M_{\mathrm{Pl}}^{2} r^{2} \Lambda_{P}\right]^{1 / 2},
\end{aligned}
$$

where

$$
\Lambda_{P} \equiv \frac{\Lambda}{P^{2}-4 M_{\mathrm{Pl}}^{2}}
$$

and $Q$ and $P$ are integration constants (with $P^{2} \neq 4 M_{\mathrm{Pl}}^{2}$ to avoid a singular geometry). The presence of the cosmological constant drastically changes the profile of the solution we analysed in section 3.1. The properties of the new configuration are the following:

- The geometry now depends on the scalar charge $P$, and the cosmological constant, through the parameter $\Lambda_{P}$ introduced in (3.13). The metric functions $f$ and $h$ differ one from the other, and the geometry appears different from (a)dS space, since the metric component $f$ scales as $r^{4}$ at large values of $r$. The profiles for $A_{0}$ and $\pi$ do not generally vanish for large values of $r$. Notice that if $Q=P=0$ the electric field controlled by $A_{0}$ is turned off, but the longitudinal profile is still non-trivial, thanks to the contribution of the cosmological constant.

- The Ricci scalar reads

$$
R=-\frac{8 \Lambda_{P}\left(15 M-5 r+4 \Lambda_{P}^{2} r^{5}\right)}{5 r\left(1+2 \Lambda_{P} r^{2}\right)^{3}} .
$$

If $\Lambda_{P}>0$, the only essential singularity is located at the origin $r=0$. If $\Lambda_{P}<0$, there is an additional essential singularity when

$$
1+2 r^{2} \Lambda_{P}=0
$$

As we will discuss in the next point, such singularity can be covered by an horizon, for appropriate choices of the parameters. The Ricci scalar vanishes at infinity; but this is not the case for all the components of the Riemann and Ricci tensor. In particular the Einstein tensor scales as $r^{2}$ at large distances, similarly to maximally symmetric space-times. It would be interesting to study how all these non-vanishing components of the curvature affect the propagation of matter and waves through space-time in our set-up.

We checked that the combination $G_{r r} A^{r}$ vanishes for our solution. This implies that the right hand side of the $r$ component of eq (2.4) vanishes: as explained in Section 2, this avoids Bekenstein's no-go arguments against the existence of black hole solutions.

- It is possible to prove that the geometry is characterised by a unique horizon, corresponding to the unique real zero of the function $f$ in (3.12a). Indeed, the quantity $r f(r)$ is a monotonic function of $r$, which is negative for $r$ very small (assuming $M>0$ ) and positive for $r$ very large (this is immediate to see if $\Lambda_{P}>0$, and simple to prove if $\Lambda_{P}<0$ ). Hence it must vanish once for some intermediate value of $r$, corresponding to the black hole horizon. As we 
have seen earlier, if $\Lambda_{P} \geq 0$ the configuration is free from essential singularities for $r \neq 0$. If $\Lambda_{P}<0$ instead we have an essential singularity at the radius

$$
r_{S}=\frac{1}{\sqrt{-2 \Lambda_{P}}} .
$$

To determine whether this singularity is covered by the black hole horizon or not, we evaluate the radial metric function $f$ at $r_{S}$. We find that the condition for ensuring that the singularity is inside the horizon is $\sqrt{-2 \Lambda_{P}} M>4 / 15$.

A change of variable

$$
\rho \equiv\left(r+\frac{2}{3} r^{3} \Lambda_{P}\right)
$$

allows us to express the metric in the following nicer form:

$$
d s^{2}=-f[r(\rho)] d t^{2}+\frac{d \rho^{2}}{f[r(\rho)]}+r^{2}(\rho) d \Omega^{2}
$$

However, since relation (3.16) can not be easily inverted, the resulting expression for $f[r(\rho)]$ is not particularly illuminating.

This concludes our discussion of the spherically symmetric configurations in our theory.

\section{Slowly rotating black hole configurations}

An open issue of screening mechanisms is to understand what happens when one renounces to spherical symmetry (see $[8,9]$ for some insights). Hence it is interesting to investigate the behaviour of stationary black hole configurations that are only axially symmetric, and not spherically symmetric. A physically relevant case are rotating black holes.

In our set-up, when turning off the cosmological constant, we are able to determine a solution for the field equations only for the case of slowly rotating configurations. A slowly rotating spacetime can be described by the metric

$$
d s^{2}=-f(r) d t^{2}+h(r)^{-1} d r^{2}+r^{2} d \theta^{2}+r^{2} \sin ^{2} \theta d \varphi^{2}+2 a r^{2} \omega(r) \sin ^{2} \theta d t d \varphi,
$$

where $a$ is a small parameter, which controls the amount of rotation. We are interested in configurations that depend at most linearly on the small parameter $a$.

We are not able to find the most general solution in this case, hence we proceed as follows. Building on the results of the previous sections, in the small $a$ limit we make the hypothesis that the geometry coincides with the small rotation limit of Kerr black holes - that is, there is not backreaction of the vector charge to the geometry. This expectation turns out to be correct, and the field equations admit a solution for the geometry corresponding to slowly rotating Kerr: $f$, $h$ correspond to the Schwarzschild geometry, while $\omega(r)$ acquires the profile $\omega=J / r^{3}$, with $J$ an integration constant parameterising angular momentum. Several components of the vector require non-trivial profiles for solving the equations. The components $A_{0}$ and $\pi$ for the time-like component of the gauge potential and longitudinal scalar $\pi$ are given by the same solutions of (3.10), (3.11) with $P=0$; the profile required for $A_{\phi}$ is

$$
A_{\phi}=a \frac{J Q}{2 M r} .
$$

This choice of vector components solves all the equations. These findings show that the geometry satisfying the field equations corresponds exactly to the slow-rotation limit of Kerr space-time (that 
by itself satisfies Einstein equation in the vacuum), while the time and angular components of the vector field coincide with the slow-rotation limit of the Kerr-Newman solution. In addition, the Abelian symmetry breaking terms switch a non-trivial profile on for the longitudinal component of the vector. Notice that the corresponding Einstein tensor vanishes, and as in the previous sections we can avoid Bekenstein no-go theorem.

\section{$5 \quad$ Higher dimensional solutions}

The system we analyzed in four dimensions can straightforwardly be analysed in higher dimensions, leading again to an analytically manageable system of equations. We consider action the (2.1) in dimensions $d=5, \ldots, 10$, with the spherically symmetric Ansatz

$$
\begin{aligned}
d s^{2} & =-f(r) d t^{2}+h(r)^{-1} d r^{2}+r^{2} d \Omega_{(d-2)}^{2}, \\
A_{\mu} & =\left(A_{0}(r), \pi(r), 0, \ldots, 0\right) .
\end{aligned}
$$

Also in different dimensions, asymptotical flatness requires a specific value for the coupling constant $\beta$, which we need to be

$$
\beta=\frac{d-3}{2 d-4} .
$$

Hence, the field solutions can be written compactly as a function of the spacetime dimension $d$ and three integration constants $M_{d}, Q_{d}, P_{d}$ as

$$
\begin{aligned}
f & =h=1-\frac{2 M_{d}}{r^{d-3}}, \\
A_{0} & =\frac{Q_{d}}{r^{d-3}}+P_{d}, \\
\pi & =\frac{\sqrt{Q_{d}^{2}+2 M_{d} P_{d}^{2} r^{d-3}+2 P_{d} Q_{d} r^{d-3}}}{r^{d-3}-2 M_{d}} .
\end{aligned}
$$

The physical properties of these configurations are similar to those in four dimensions, thus we conjecture that these solution should exists in dimensions $d>10$, with the same formulas given above.

\section{Discussion}

We examined exact black hole solutions for a particular vector-tensor theory of gravity, with the Abelian symmetry broken by a certain non-minimal coupling of the vector $A^{\mu}$ to gravity, given by

$$
\beta G_{\mu \nu} A^{\mu} A^{\nu}
$$

where $G_{\mu \nu}$ is the Einstein tensor. Our solutions are characterised by a long range electric field associated with an electric charge $Q$, and by a non-trivial profile for the vector's longitudinal polarisation, associated with a charge $P$ which however does not contribute to a Gauss law. We construct these solutions in four and higher dimensions, and find that asymptotical flatness, in the absence of a bare cosmological constant, singles out a particular value for above's coupling $\beta$, given by $\beta=(d-3) /(2 d-4)$, with $d$ the number of dimensions. By choosing this value, we have been able to integrate explicitly all the equations of motion, and found the most general solution, that can be simply extended to include a bare cosmological constant in $4 \mathrm{~d}$. Moreover, we determined exact solutions of the field equations for slowly rotating black holes. The final expressions for the field configurations are relatively simple, and we discussed their physical properties and implications. 
We also discussed in some detail how our system is able to avoid Bekenstein's no-go arguments [20] on black holes with massive vector hairs.

It would be important to check in detail whether our configurations are stable under small disturbances, possibly implementing the techniques that have been recently developed for studying similar scalar-tensor black hole configurations [35]. We explicitly checked that our solutions are stable under spherically symmetric fluctuations in certain cases, but a more general analysis would be needed to settle the question.

It would also be interesting to explore solutions which are not asymptotically flat, for which $\beta \neq(d-3) /(2 d-4)$, or to consider more general theories that break the Abelian symmetry without intoducing a ghost, as the ones considered here. Actually, we have looked into some of these more general theories, but have not been able to find analytical solutions. However a numerical analysis of these systems might be feasible.

Finally, it would also be very interesting to investigate whether these configurations can have some astrophysical relevance. It is important to study realistic black hole formation in this scenarios, and see whether they can lead to configurations equipped by a long distance scalar hair associated with the vector longitudinal polarization. Moreover, our Abelian symmetry breaking coupling of vector to gravity is extremely suppressed for weakly gravitating systems, hence such terms might realistically describe a coupling between electromagnetism and gravity, with potentially distinctive effects in the strong gravity regimes involving black holes and cosmology.

\section{Acknowledgments}

It is a pleasure to thank Ivonne Zavala for discussions. GT is partially supported by STFC grant ST/N001435/1. GN and, partially, JC are supported by the grant CONACYT/179208. JC is also supported by the grant CONACYT/263819.

\section{References}

[1] B. P. Abbott et al. [LIGO Scientific and Virgo Collaborations], Phys. Rev. Lett. 116 (2016) 6, 061102 doi:10.1103/PhysRevLett.116.061102 [arXiv:1602.03837 [gr-qc]].

[2] C. A. R. Herdeiro and E. Radu, Int. J. Mod. Phys. D 24 (2015) 09, 1542014 doi:10.1142/S0218271815420146 [arXiv:1504.08209 [gr-qc]].

[3] T. Clifton, P. G. Ferreira, A. Padilla and C. Skordis, Phys. Rept. 513 (2012) 1 doi:10.1016/j.physrep.2012.01.001 [arXiv:1106.2476 [astro-ph.CO]].

[4] J. Khoury, arXiv:1312.2006 [astro-ph.CO].

[5] A. I. Vainshtein, Phys. Lett. B 39, 393 (1972). doi:10.1016/0370-2693(72)90147-5

[6] E. Babichev and C. Deffayet, Class. Quant. Grav. 30, 184001 (2013) doi:10.1088/0264-9381/30/18/184001 [arXiv:1304.7240 [gr-qc]].

[7] K. Koyama, G. Niz and G. Tasinato, Phys. Rev. D 88, 021502 (2013) doi:10.1103/PhysRevD.88.021502 [arXiv:1305.0279 [hep-th]].

[8] J. Chagoya, K. Koyama, G. Niz and G. Tasinato, JCAP 1410 (2014) 10, 055 doi:10.1088/1475-7516/2014/10/055 [arXiv:1407.7744 [hep-th]].

[9] J. K. Bloomfield, C. Burrage and A. C. Davis, Phys. Rev. D 91, no. 8, 083510 (2015) doi:10.1103/PhysRevD.91.083510 [arXiv:1408.4759 [gr-qc]].

[10] C. Burrage, E. J. Copeland and J. Stevenson, Phys. Rev. D 91, 065030 (2015) doi:10.1103/PhysRevD.91.065030 [arXiv:1412.6373 [hep-th]]. 
[11] K. Koyama and J. Sakstein, Phys. Rev. D 91 (2015) 124066 doi:10.1103/PhysRevD.91.124066 [arXiv:1502.06872 [astro-ph.CO]];

K. Koyama, arXiv:1504.04623 [astro-ph.CO].

[12] L. Hui and A. Nicolis, Phys. Rev. Lett. 110 (2013) 241104 doi:10.1103/PhysRevLett.110.241104 [arXiv:1202.1296 [hep-th]].

[13] M. Rinaldi, Phys. Rev. D 86 (2012) 084048 doi:10.1103/PhysRevD.86.084048 [arXiv:1208.0103 $[\mathrm{gr}-\mathrm{qc}]]$.

[14] T. P. Sotiriou and S. Y. Zhou, Phys. Rev. D 90 (2014) 124063 [Phys. Rev. D 90 (2014) 12] doi:10.1103/PhysRevD.90.124063 [arXiv:1408.1698 [gr-qc]].

[15] E. Babichev and C. Charmousis, JHEP 1408 (2014) 106 doi:10.1007/JHEP08(2014)106 [arXiv:1312.3204 [gr-qc]].

[16] T. P. Sotiriou, Class. Quant. Grav. 32 (2015) 21, 214002 doi:10.1088/0264-9381/32/21/214002 [arXiv:1505.00248 [gr-qc]].

[17] M. S. Volkov, arXiv:1601.08230 [gr-qc].

[18] C. Herdeiro and E. Radu, Class. Quant. Grav. 32, no. 14, 144001 (2015) doi:10.1088/0264-9381/32/14/144001 [arXiv:1501.04319 [gr-qc]].

[19] H. O. Silva, A. Maselli, M. Minamitsuji and E. Berti, arXiv:1602.05997 [gr-qc].

[20] J. D. Bekenstein, Phys. Rev. D 5, 1239 (1972). doi:10.1103/PhysRevD.5.1239

[21] J. D. Bekenstein, Phys. Rev. Lett. 28 (1972) 452. doi:10.1103/PhysRevLett.28.452

[22] J. D. Bekenstein, Phys. Rev. D 5 (1972) 2403. doi:10.1103/PhysRevD.5.2403

[23] S. L. Adler and R. B. Pearson, Phys. Rev. D 18 (1978) 2798. doi:10.1103/PhysRevD.18.2798

[24] G. Tasinato, JHEP 1404 (2014) 067 [arXiv:1402.6450 [hep-th]].

[25] B. M. Gripaios, JHEP 0410, 069 (2004) [hep-th/0408127].

[26] L. Heisenberg, arXiv:1402.7026 [hep-th].

[27] A. De Felice, L. Heisenberg, R. Kase, S. Tsujikawa, Y. 1. Zhang and G. B. Zhao, arXiv:1602.00371 [gr-qc];

[28] G. Tasinato, Class. Quant. Grav. 31 (2014) 225004 [arXiv:1404.4883 [hep-th]];

M. Hull, K. Koyama and G. Tasinato, JHEP 1503 (2015) 154 [arXiv:1408.6871 [hep-th]];

J. Chagoya and G. Tasinato, arXiv:1511.07805 [hep-th].

N. Khosravi, Phys. Rev. D 89 (2014) 12, 124027 doi:10.1103/PhysRevD.89.124027 [arXiv:1404.7503 [hep-th]];

F. Charmchi, Z. Haghani, S. Shahidi and L. Shahkarami, arXiv:1511.07034 [hep-th];

E. Allys, P. Peter and Y. Rodriguez, JCAP 1602, no. 02, 004 (2016)

doi:10.1088/1475-7516/2016/02/004 [arXiv:1511.03101 [hep-th]];

J. B. Jimnez and L. Heisenberg, arXiv:1602.03410 [hep-th];

J. Beltran Jimenez and A. L. Maroto, JCAP 1012 (2010) 025 doi:10.1088/1475-7516/2010/12/025 [arXiv:1010.4513 [astro-ph.CO]];

J. Beltrn Jimnez and T. S. Koivisto, Class. Quant. Grav. 31 (2014) 135002

doi:10.1088/0264-9381/31/13/135002 [arXiv:1402.1846 [gr-qc]];

J. B. Jimnez and T. S. Koivisto, arXiv:1509.02476 [gr-qc].

[29] M. Hull, K. Koyama and G. Tasinato, arXiv:1510.07029 [hep-th];

[30] M. S. Turner and L. M. Widrow, Phys. Rev. D 37 (1988) 2743. doi:10.1103/PhysRevD.37.2743

[31] A. Golovnev, V. Mukhanov and V. Vanchurin, JCAP 0806 (2008) 009 doi:10.1088/1475-7516/2008/06/009 [arXiv:0802.2068 [astro-ph]]. 
[32] E. Ayon-Beato, [gr-qc/0210001].

[33] D. W. Pang, JHEP 1001 (2010) 116 doi:10.1007/JHEP01(2010)116 [arXiv:0911.2777 [hep-th]].

[34] S. M. Carroll, San Francisco, USA: Addison-Wesley (2004) 513 p

[35] H. Ogawa, T. Kobayashi and T. Suyama, arXiv:1510.07400 [gr-qc];

K. Takahashi, T. Suyama and T. Kobayashi, arXiv:1511.06083 [gr-qc];

A. Cisterna, M. Cruz, T. Delsate and J. Saavedra, Phys. Rev. D 92 (2015) 10, 104018 doi:10.1103/PhysRevD.92.104018 [arXiv:1508.06413 [gr-qc]].

A. Anabalon, J. Bičák and J. Saavedra, Phys. Rev. D 90 (2014) 12, 124055

doi:10.1103/PhysRevD.90.124055 [arXiv:1405.7893 [gr-qc]].

T. Kobayashi, H. Motohashi and T. Suyama, Phys. Rev. D 89 (2014) 8, 084042

doi:10.1103/PhysRevD.89.084042 [arXiv:1402.6740 [gr-qc]]. 